\title{
Supplementation with Human-Origin Probiotic Lactobacillus plantarum PL-02 Could Effectively Improve Muscle Mass and Exercise Performance, and Have an Anti-Fatigue Effect
}

\author{
Mon-Chien Lee \\ National Taiwan Sport University \\ Yi-Ju Hsu \\ National Taiwan Sport University \\ Hsieh-Hsun Ho \\ Bioflag Biotech Co, Ltd \\ Yi-Wei Kuo \\ Bioflag Biotech Co, Ltd \\ Wen-Yang Lin \\ Bioflag Biotech Co, Ltd \\ Shin-Yu Tsai \\ Bioflag Biotech Co, Ltd \\ Wei-Ling Chen \\ National Taiwan Sport University \\ Che-Li Lin
}

Taipei Medical University

Chi-Chang Huang ( $\sim$ john5523@ntsu.edu.tw )

National Taiwan Sport University

\section{Research Article}

Keywords: Lactobacillus plantarum, gut microbiota, muscle mass, exercise performance, fatigue

Posted Date: June 1st, 2021

DOI: https://doi.org/10.21203/rs.3.rs-558635/v1

License: () (1) This work is licensed under a Creative Commons Attribution 4.0 International License.

Read Full License 


\section{Abstract}

Gut microbiota are very important for energy metabolism and regulation, which in turn affect the health and physiological functions of the host. The most direct way to change the gut microbiota is to supplement with probiotics. In this study, we screened Lactobacillus plantarum (PL-02), a probiotic of human-origin, from the intestines of Olympic gold medalists and explored the role of PL-02 in improved exercise endurance performance, reduced fatigue biochemical parameters, and changes in body composition. Male Institute of Cancer Research (ICR) mice were assigned to $0 \mathrm{CFU} / \mathrm{kg}$ (vehicle), $2.05 \times$ $10^{9} \mathrm{CFU} / \mathrm{kg}(\mathrm{PL}-02-1 \mathrm{X}), 4.10 \times 10^{9} \mathrm{CFU} / \mathrm{kg}$ (PL-02-2X), and $1.03 \times 10^{10} \mathrm{CFU} / \mathrm{kg}$ (PL-02-5X) groups and were fed by oral gavage once daily for 4 weeks to assess exercise performance, fatigue parameters, and body composition. The results showed that 4 weeks of PL-02 supplementation could significantly increase muscle mass, improve muscle strength and endurance performance, and increase hepatic and muscular glycogen storage. Furthermore, PL-02 could significantly decrease fatigue biochemical parameters, such as lactate, blood urea nitrogen (BUN), ammonia, and creatine kinase (CK) levels, after exercise. We believe that PL-02 can be used as a supplement to improve exercise performance and for its anti-fatigue effect.

\section{Introduction}

Exercise-induced fatigue can be roughly divided into central fatigue and peripheral fatigue, which is a common and complex multi-dimensional symptom ${ }^{1}$. Among them, peripheral fatigue generally refers to long-term or high-intensity exercise; the body is unable to provide or maintain the required energy load, resulting in decreased performance and producing fatigue ${ }^{2}$. During exercise, glycogen from the liver and muscles is metabolized into glucose by the phosphocreatine system, and glucose is further metabolized to meet higher energy requirements ${ }^{3}$. When the energy consumed exceeds the supply demand, the byproducts that cause fatigue will increase, especially in the muscles ${ }^{4}$. Due to the consumption of creatine phosphate, the accumulation of neuromuscular signaling implants, glycogen, and internal metabolites (including lactic acid, inorganic phosphorus, and ammonia), it is difficult for the muscles to maintain continuous contraction ${ }^{4,5}$. These metabolites can cause muscle fatigue through intracellular acidosis in the body ${ }^{6}$. Therefore, to recover from fatigue caused by exercise, it is necessary to repair damage to the body and eliminate the metabolites accumulated from exercise. In addition to scientific training methods and nutritional supplementation for those who engage in sports, in recent years, views on gut microbiota relative to energy regulation have gradually attracted attention ${ }^{7}$.

The gut microbiota contains trillions of different microbial communities. Its rich and diverse ecosystem interacts with the host, which is the key to metabolism and is believed to play a key role in the overall health and disease of the host ${ }^{8}$. By promoting the development and maturation of intestinal epithelial cells, it activates the intestinal barrier function and prevents the colonization of pathogenic bacteria to maintain the health of the host ${ }^{9}$. The most important source of nutrition for the gut microbiota is indigestible carbohydrates in the diet ${ }^{10}$. Promoting the digestion, absorption, and fermentation of them to 
produce short-chain fatty acids (SCFA), such as acetate, propionate, and butyrate, as well as $\mathrm{H} 2$ and $\mathrm{CO} 2$ gases, ammonia, amines, phenols, and energy, helps to maintain cell function ${ }^{11}$. Among them, propionic acid is used in gluconeogenic liver cells, while butyrate is affected by the acetoacetyl-CoA produced by fatty acid $\beta$-oxidation (FAO), which enters the trichloroacetic acid cycle to produce adenosine triphosphate (ATP) and $\mathrm{CO}_{2}{ }^{12}$. In addition to the induction of PGC-1a gene expression in skeletal muscle and brown adipose tissue (BAT) $)^{13}$ and the activation of the activated protein kinase-acetyl-CoA carboxylase (AMPK-ACC) pathway to improve respiratory capacity, FAO is a key regulator of energy production and mitochondrial function. It is regarded as one of the important factors for improving exercise performance ${ }^{14}$. In recent years, a study has shown that through the action of the intestinal axis, gut microbiota seems to have an impact on muscle mass ${ }^{15}$.

There are many factors that affect changes in the gut microbiota, including age, gender, antibiotics, diet, and exercise ${ }^{16}$. However, supplementing with probiotics is the fastest and most effective method. Through ingestion, it can be colonized in the intestinal tract to increase the diversity and richness ratio of good bacteria in the intestine, thereby increasing the host's energy metabolism and promoting health ${ }^{17}$. Different strains have different effects, and the most of the common probiotics are Bifidobacterium or Lactobacillus $^{18}$. Among them, Lactobacillus plantarum (L. plantarum) is a Gram-positive species and belongs to the Lactobacillus spp. Many studies in the past have shown that it has anti-inflammatory ${ }^{19}$, antioxidant ${ }^{20}$, and other effects. In addition, L. plantarum can help bacteria use lactic acid, firstly producing acetyl-CoA from lactic acid. Two molecules of acetyl-CoA are combined and reduced to produce butyryl-CoA, then converts butyryl-CoA to butyryl-phosphate by using the phosphotransbutyrylase enzyme. Butyryl-phosphate is converted into butyrate by using butyrate kinase and releases ATP, is regarded as an important factor in reducing exercise fatigue ${ }^{21}$. Although it has been confirmed in animal ${ }^{22}$ and human ${ }^{23}$ experiments that $L$. plantarum could improve exercise performance and reduce fatigue biochemical indicators, the strain was selected from kimchi.

In the current study, we screened L. plantarum (PL-02), which is a human-origin probiotic, from the intestines of Olympic gold medalists and explored the effect of PL-02 in terms of improving exercise endurance, reducing fatigue biochemical parameters, and changing body composition. In addition, we further analyzed the gut microbiota, hoping to explore the possible mechanism of probiotics against fatigue and to confirm its safety.

\section{Materials And Methods}

\section{Sample preparation}

We screened Lactobacillus plantarum (PL-02) from the gut microbiota of women's Olympic weightlifting gold medalists. The Food Industry Research and Development Institute (Hsinchu, Taiwan) has confirmed that the isolate is Lactobacillus plantarum. The dry product of PL-02 was prepared and provided by 
Bioflag Biotech Co, Ltd. (Tainan, Taiwan). The viable number of PL-02 cells was $1.07 \times 10^{11} \mathrm{CFU} / \mathrm{g}$. Before consumption, the powder was suspended in phosphate buffered saline (PBS, pH 7.2).

\section{Animals and experimental design}

In the current study, the microbial strain was isolated from one of the Co-authors (Wei-Ling Chen) of the Manuscript, all experimental used the animal model for research, and the methods followed the Guideline for the Care and Use of Laboratory Animals of Council of Agriculture, Executive Yuan, Taiwan and ARRIVE guidelines. The experiments were approved by the Institutional Animal Care and Use Committee (IACUC) of National Taiwan Sport University (approval number: IACUC-10904). All the mice were kept in a lightdark cycle for 12 hours at room temperature $\left(23 \pm 2^{\circ} \mathrm{C}\right)$ and $50-60 \%$ humidity and were provided with reverse osmosis (RO) water and a standard daily diet (No. 5001; PMI Nutrition International, Brentwood, MO, USA) ad libitum. Since probiotics are used in humans at a dose of $1 \times 10^{10}$ live bacteria per day ${ }^{24}$, the human body surface area is converted from mouse equivalent dose (HED) to a mouse dose. Forty 6week-old male ICR mice were purchased from BioLASCO (Yi-Lan, Taiwan). After a 2-week acclimation period, all the mice were divided into four groups ( $n=10$ in each group), and they were administered by gavage once a day for 4 weeks: 1): vehicle group (0 CFU/kg); 2): PL-02-1X group (2.05 × $\left.10^{9} \mathrm{CFU} / \mathrm{kg}\right) ; 3$ ): $\mathrm{PL-02-2X}$ group $\left(4.10 \times 10^{9} \mathrm{CFU} / \mathrm{kg}\right)$, and 4): PL-02-5X group $\left(1.03 \times 10^{10} \mathrm{CFU} / \mathrm{kg}\right)$. All groups were given the same volume of PBS or supplements, and the dose of PL-02 was determined according to the body weight of each mouse.

\section{Forelimb grip strength}

As previously described, a low-force test system (Model-RX-5, Aikoh Engineering, Nagoya, Japan) with a tension rod (diameter $2 \mathrm{~mm}$, length $7.5 \mathrm{~cm}$ ) and a force sensor was used to measure the grip of mice ${ }^{25}$.

\section{Exercise performance test}

In order to understand the effect of PL-02 on improving exercise endurance performance, we conducted a swimming exhaustion test. The tails of all mice are attached with $5 \%$ body weight, and the duration of such swimming was recorded. The test mice are then forced to swim until they lose coordinated movement or cannot return to the surface within $7 \mathrm{~s}$, as described previously 26 .

\section{Determination of fatigue-associated biochemical variables}

In order to understand the effect of PL-02 supplementation on fatigue-related indicators and physiological adaptation after exercise, we collected blood samples after swimming for 10 minutes and resting for 20 minutes to analyze blood lactic acid, blood ammonia, and glucose. After 90 minutes of prolonged exercise and 60 minutes of rest, we immediately evaluated other variables such as blood urine nitrogen (BUN) and creatine kinase (CK). All the mice were ensured for fasting before all exercise tests to ensure conditional control, as previously described ${ }^{26}$. The sample was centrifuged at $1500 \times \mathrm{g}$ at $4^{\circ} \mathrm{C}$ for 10 minutes, and the serum was collected and measured by an automatic analyzer (Hitachi, Tokyo, Japan, Hitachi 7060). 


\section{Clinical biochemical profiles}

At the end of the study, all the mice were euthanized by $95 \%$ CO2 asphyxiation one hour after the last treatment and blood was obtained by cardiac puncture. Serum was collected after centrifugation, and biochemical indexes were assessed by Hitachi 7060 autoanalyzer. Levels of glucose (GLU), albumin (ALB), total protein (TP), creatine kinase (CK), aspartate aminotransferase (AST), alanine transaminase (ALT), total cholesterol (TC), triglycerides (TG), blood urea nitrogen (BUN), creatinine (CREA), and uric acid (UA) were measured.

\section{Body composition, glycogen content, and histopathology}

After the mice were euthanized, the liver, muscle, kidney, heart, lung, epididymal fat pad (EFP), and brown adipose tissue (BAT) were accurately excised and weighed. The organs were carefully removed, chopped, and fixed in $10 \%$ formalin. The tissue was embedded in paraffin and cut into $4 \mu \mathrm{m}$-thick sections for morphological and pathological evaluation. As mentioned earlier, the sections were stained with hematoxylin and eosin (H\&E) and inspected under an optical microscope equipped with a charge-coupled device (CCD) camera (BX-51, Olympus, Tokyo, Japan) ${ }^{25}$. Parts of the muscle and liver tissues were stored in liquid nitrogen for glycogen content analysis, as previously described ${ }^{27}$.

\section{Bacterial DNA extraction and 16S rRNA sequencing}

According to the method previously used in our laboratory, immediately after the euthanasia of the mice, the collected samples were stored at $-80^{\circ} \mathrm{C}$ for DNA extraction. The detailed procedures for sample extraction, preparation, and analysis have been described previously ${ }^{28}$.

\section{Statistical analysis}

All data are expressed as mean \pm SD. The statistical analysis was performed in SAS 9.0 (SAS Inst., Cary, NC, USA). Using Duncan's post hoc test, multiple group comparisons were analyzed by one-way analysis of variance (ANOVA). Cochran-Armitage test is used for dose-effect trend analysis. Statistical significance was set at $p<0.05$.

\section{Results}

\section{General characteristics of mice with PL-02 supplementation for four weeks}

As shown on Table 1, after four consecutive weeks of supplementation with PL-02, the weight of each group of mice showed stable growth; there was no significant difference between the groups, and there were no significant differences in the food intake and water intake of each group of mice. However, supplementation with PL-02 could effectively only improve skeletal muscle mass, which in the vehicle, PL-02-1X, PL-02-2X, and PL-02-5X groups was $0.37 \pm 0.04,0.39 \pm 0.02,0.40 \pm 0.03$, and $0.41 \pm 0.02(\mathrm{~g})$, respectively. Compared with the placebo group, only the PL-02-5X group significantly increased by 1.10 fold $(p=0.0028)$. But there is still a significant dose dependence, $p=0.0015$. As the tissue weight is 
affected by the weight difference, we divided the tissue weight by the relative percentage of body weight and found that the relative skeletal muscles weight in the vehicle, PL-02-1X, PL-02-2X, and PL-02-5X groups was $1.00 \pm 0.04,1.05 \pm 0.02,1.06 \pm 0.03$, and $1.10 \pm 0.02(\mathrm{~g})$, respectively. Compared with the placebo group, the PL-02-1X, PL-02-2X, and PL-02-5X groups were significantly increased relative skeletal muscles weight by 1.05 -fold $(p=0.0014), 1.06$-fold $(p=0.0001), 1.11$-fold $(p<0.0001)$, respectively. The effect of PL-02 supplementation on relative muscle weight was dose dependent $(p<0.0001)$. 
Table 1

Effect of PL-02 supplementation on various parameters.

\begin{tabular}{|c|c|c|c|c|c|}
\hline Characteristic & $\begin{array}{l}\text { Vehicle } \\
\text { (PBS) }\end{array}$ & PL-02-1X & PL-02-2X & PL-02-5X & $\begin{array}{l}\text { Trend } \\
\text { Analysis }\end{array}$ \\
\hline Initial BW (g) & $34.8 \pm 1.1$ & $34.6 \pm 1.3$ & $34.5 \pm 1.4$ & $34.6 \pm 1.0$ & 0.4507 \\
\hline Final BW (g) & $37.3 \pm 2.8$ & $37.5 \pm 1.4$ & $37.4 \pm 1.4$ & $37.2 \pm 1.6$ & 0.8624 \\
\hline $\begin{array}{l}\text { Water intake } \\
\text { (mL/mouse/day) }\end{array}$ & $8.1 \pm 2.3$ & $8.1 \pm 2.2$ & $8.2 \pm 2.3$ & $8.1 \pm 1.4$ & 0.2061 \\
\hline $\begin{array}{l}\text { Food intake } \\
\text { (g/mouse/day) }\end{array}$ & $6.8 \pm 1.3$ & $6.7 \pm 1.3$ & $6.8 \pm 1.3$ & $6.4 \pm 1.2$ & 0.1453 \\
\hline Liver (g) & $2.01 \pm 0.12$ & $1.99 \pm 0.16$ & $2.00 \pm 0.18$ & $\begin{array}{l}2.01 \pm \\
0.12\end{array}$ & 0.8113 \\
\hline Muscle (g) & $\begin{array}{l}0.37 \pm 0.04 \\
a\end{array}$ & $\begin{array}{l}0.39 \pm 0.02 \\
\mathrm{ab}\end{array}$ & $\begin{array}{l}0.40 \pm 0.03 \\
\mathrm{ab}\end{array}$ & $\begin{array}{l}0.41 \pm \\
0.02^{b}\end{array}$ & $0.0015^{*}$ \\
\hline Quadriceps (g) & $0.51 \pm 0.06$ & $0.53 \pm 0.06$ & $0.53 \pm 0.04$ & $\begin{array}{l}0.53 \pm \\
0.05\end{array}$ & 0.5256 \\
\hline Kidney (g) & $0.59 \pm 0.07$ & $0.60 \pm 0.06$ & $0.59 \pm 0.05$ & $\begin{array}{l}0.59 \pm \\
0.03\end{array}$ & 0.9523 \\
\hline Heart (g) & $0.19 \pm 0.03$ & $0.20 \pm 0.02$ & $0.20 \pm 0.03$ & $\begin{array}{l}0.19 \pm \\
0.02\end{array}$ & 0.2786 \\
\hline Lung (g) & $0.25 \pm 0.03$ & $0.24 \pm 0.05$ & $0.25 \pm 0.01$ & $\begin{array}{l}0.24 \pm \\
0.03\end{array}$ & 0.6396 \\
\hline $\operatorname{EFP}(\mathrm{g})$ & $0.36 \pm 0.04$ & $0.35 \pm 0.10$ & $0.36 \pm 0.15$ & $\begin{array}{l}0.34 \pm \\
0.12\end{array}$ & 0.3592 \\
\hline BAT (g) & $0.11 \pm 0.02$ & $0.11 \pm 0.02$ & $0.11 \pm 0.03$ & $\begin{array}{l}0.10 \pm \\
0.01\end{array}$ & 0.5523 \\
\hline Cecum (g) & $0.83 \pm 0.08$ & $0.83 \pm 0.16$ & $0.84 \pm 0.08$ & $\begin{array}{l}0.84 \pm \\
0.09\end{array}$ & 0.6607 \\
\hline *Relative liver weight (\%) & $5.39 \pm 0.14$ & $5.31 \pm 0.25$ & $5.35 \pm 0.31$ & $\begin{array}{l}5.40 \pm \\
0.13\end{array}$ & 0.9568 \\
\hline $\begin{array}{l}\text { Relative muscle weight } \\
(\%)\end{array}$ & $\begin{array}{l}1.00 \pm 0.04 \\
a\end{array}$ & $\begin{array}{l}1.05 \pm 0.02 \\
b\end{array}$ & $\begin{array}{l}1.06 \pm 0.03 \\
b\end{array}$ & $\begin{array}{l}1.10 \pm \\
0.02^{c}\end{array}$ & $<0.0001^{\star}$ \\
\hline $\begin{array}{l}\text { Relative quadriceps } \\
\text { weight (\%) }\end{array}$ & $1.37 \pm 0.06$ & $1.40 \pm 0.11$ & $1.41 \pm 0.05$ & $\begin{array}{l}1.43 \pm \\
0.06\end{array}$ & 0.0785 \\
\hline Relative kidney weight (\%) & $1.58 \pm 0.07$ & $1.60 \pm 0.11$ & $1.58 \pm 0.08$ & $\begin{array}{l}1.58 \pm \\
0.02\end{array}$ & 0.8170 \\
\hline
\end{tabular}

Data are expressed as mean \pm SD ( $n=10$ mice per group). EFP: epididymal fat pad; BAT: brown adipose tissue. *Relative to body weight. 


\begin{tabular}{|llllll|}
\hline Characteristic & $\begin{array}{l}\text { Vehicle } \\
\text { (PBS) }\end{array}$ & PL-02-1X & PL-02-2X & PL-02-5X & $\begin{array}{l}\text { Trend } \\
\text { Analysis }\end{array}$ \\
\hline Relative heart weight (\%) & $0.51 \pm 0.04$ & $0.52 \pm 0.03$ & $0.53 \pm 0.05$ & $0.52 \pm$ & 0.1083 \\
& & & & 0.02 & \\
\hline Relative lung weight (\%) & $0.66 \pm 0.04$ & $0.63 \pm 0.12$ & $0.67 \pm 0.02$ & $0.64 \pm$ & 0.5418 \\
& & & & 0.06 & \\
\hline Relative EFP weight (\%) & $0.96 \pm 0.05$ & $0.94 \pm 0.23$ & $0.96 \pm 0.37$ & $0.91 \pm$ & 0.2944 \\
& & & & 0.29 & \\
\hline Relative BAT weight (\%) & $0.28 \pm 0.05$ & $0.30 \pm 0.04$ & $0.29 \pm 0.02$ & $0.27 \pm$ & 0.4544 \\
& & & & 0.03 & \\
\hline Relative cecum weight (\%) & $2.21 \pm 0.07$ & $2.21 \pm 0.35$ & $2.25 \pm 0.15$ & $2.25 \pm$ & 0.6468 \\
& & & & 0.14 & \\
\hline
\end{tabular}

Data are expressed as mean \pm SD ( $n=10$ mice per group). EFP: epididymal fat pad; BAT: brown adipose tissue. *Relative to body weight.

\section{Effect of PL-02 supplementation on exercise performance}

On grip strength, after 4 weeks of PL-02 supplementation, the mean forelimb grip strength so mice in the vehicle, PL-02-1X, PL-02-2X, and PL-02-5X groups were $126 \pm 7,141 \pm 8,147 \pm 6$, and $151 \pm 6 \mathrm{~g}$, respectively (Fig. 1a) The PL-02-1X, PL-02-2X, and PL-02-5X groups were significantly higher than the vehicle group by 1.11 -fold $(p<0.0001), 1.16$-fold $(p<0.0001)$, and 1.20 -fold $(p<0.0001)$, respectively. Relative grip strength (\%), normalized to body weight, was also significantly higher in groups with PL-02 supplementation (Fig. 1b). The effects of PL-02 supplementation on absolute and relative grip strength were dose dependent $(p<0.0001)$.

After four weeks of PL-02 supplementation, the exhaustive swim times in the vehicle, PL-02-1X, PL-02-2X, and PL-02-5X groups were $6.81 \pm 1.04,8.16 \pm 0.75,9.74 \pm 0.93$, and $17.16 \pm 1.53 \mathrm{~min}$, respectively. The average exhaustive swim time of the PL-02-1X, PL-02-2X, and PL-02-5X groups was significantly increased by 1.20 -fold $(p=0.0092), 1.43$-fold $(p<0.0001)$, and 2.52-fold $(p<0.0001)$, respectively, as compared to the vehicle group. The trend analyses showed that the effect of PL-02 supplementation on maximum swim time was dose dependent ( $p<0.0001)$ (Fig. 1c).

\section{Effect of PL-02 supplementation on serum lactate levels after the 10-min swim test}

After 4 weeks of supplementation with PL-02, all the mice were subjected to the 10 -min swimming test to evaluate the levels of lactate (Table 2). Before swimming, there was no significant difference in blood lactate levels between groups. The serum lactate levels of mice in the vehicle, PL-02-1X, PL-02-2X, and PL-02-5X groups were $7.78 \pm 0.70,5.67 \pm 0.78,4.90 \pm 0.55$ and $4.65 \pm 0.52 \mathrm{mmol} / \mathrm{L}$ after $10 \mathrm{~min}$ of swimming, respectively. Compared with the placebo group, the PL-02-1X, PL-02-2X, and PL-02-5X groups were significantly decreased by $27.16 \%(p<0.0001), 37.05 \%(p<0.0001)$, and $40.26 \%(p<0.0001)$, respectively. Based on the serum lactate concentration before and after $10 \mathrm{~min}$ of swimming, the lactate 
production rates were determined to be $2.51 \pm 0.22,1.80 \pm 0.08,1.55 \pm 0.09$, and $1.46 \pm 0.09$, respectively, in the vehicle, PL-02-1X, PL-02-2X, and PL-02-5X groups. The PL-02-1X, PL-02-2X, and PL-02-5X groups were significantly lower than vehicle group by $27.99 \% \%(p<0.0001), 38.26 \%(p<0.0001)$, and $41.74 \%$ $(p<0.0001)$, respectively.

Table 2

Effect of PL-02 on lactate levels.

\begin{tabular}{|c|c|c|c|c|c|}
\hline \multirow[t]{2}{*}{ Time point } & Vehicle & PL-02-1X & PL-02-2X & PL-02-5X & $\begin{array}{l}\text { Trend } \\
\text { Analysis }\end{array}$ \\
\hline & \multicolumn{5}{|c|}{ Lactate (mmol/L) } \\
\hline Before swimming (A) & $3.15 \pm 0.54$ & $3.13 \pm 0.36$ & $3.17 \pm 0.33$ & $3.19 \pm 0.32$ & 0.6067 \\
\hline After swimming (B) & $\begin{array}{l}7.78 \pm 0.70 \\
c\end{array}$ & $\begin{array}{l}5.67 \pm 0.78 \\
b\end{array}$ & $\begin{array}{l}4.90 \pm 0.55 \\
a\end{array}$ & $\begin{array}{l}4.65 \pm 0.52 \\
a\end{array}$ & $<0.0001^{*}$ \\
\hline \multirow[t]{2}{*}{ After a 20 min rest (C) } & $\begin{array}{l}6.30 \pm 0.56 \\
c\end{array}$ & $\begin{array}{l}4.58 \pm 0.54 \\
b\end{array}$ & $\begin{array}{l}3.87 \pm 0.42 \\
a\end{array}$ & $\begin{array}{l}3.46 \pm 0.34 \\
\mathrm{a}\end{array}$ & $<0.0001^{*}$ \\
\hline & \multicolumn{5}{|c|}{ Rate of lactate production and clearance } \\
\hline Production rate $=\mathrm{B} / \mathrm{A}$ & $\begin{array}{l}2.51 \pm 0.22 \\
c\end{array}$ & $\begin{array}{l}1.80 \pm 0.08 \\
b\end{array}$ & $\begin{array}{l}1.55 \pm 0.09 \\
a\end{array}$ & $\begin{array}{l}1.46 \pm 0.09 \\
\mathrm{a}\end{array}$ & $<0.0001^{*}$ \\
\hline $\begin{array}{l}\text { Clearance rate }=(B- \\
\text { C) } / B\end{array}$ & $\begin{array}{l}0.19 \pm 0.04 \\
\mathrm{a}\end{array}$ & $\begin{array}{l}0.19 \pm 0.04 \\
a\end{array}$ & $\begin{array}{l}0.21 \pm 0.04 \\
\mathrm{a}\end{array}$ & $\begin{array}{l}0.25 \pm 0.03 \\
b\end{array}$ & $<0.0001^{*}$ \\
\hline \multicolumn{6}{|c|}{$\begin{array}{l}\text { Lactate production rate }(B / A) \text { was the value of the lactate level after exercise }(B) \text { divided by that } \\
\text { before exercise }(A) \text {. Clearance rate }(B-C) / B \text { was defined as lactate level after swimming }(B) \text { minus } \\
\text { that after } 20 \text { min rest }(C) \text { divided by that after swimming }(B) \text {. Data are expressed as mean } \pm S D(n=10 \\
\text { mice per group). Values in the same row with different superscript letters }(a, b, c, d) \text { differ significantly } \\
p<0.05 \text {. }\end{array}$} \\
\hline
\end{tabular}

After 20 min rest following the swimming test, the blood lactate levels in the vehicle, PL-02-1X, PL-02-2X, and PL-02-5X groups were $6.30 \pm 0.56,4.58 \pm 0.54,3.87 \pm 0.42$, and $3.46 \pm 0.34 \mathrm{mmol} / \mathrm{L}$, respectively. This represented a decrease of $27.35 \%(p=0.0002), 38.61 \%(p<0.0001)$, and $45.12 \%(p<0.0001)$ in the PL-02$1 \mathrm{X}, \mathrm{PL}-02-2 \mathrm{X}$, and PL-02-5X groups, respectively, compared to the vehicle group. The clearance rate is used to understand the recovery effect of lactate after 10 minutes of exercise then 20 minutes of rest, which was determined to be $0.19 \pm 0.04,0.19 \pm 0.04,0.21 \pm 0.04$, and $0.25 \pm 0.03$, respectively, in the vehicle, PL-02-1X, PL-02-2X, and PL-02-5X groups. Compared with the placebo group, only the PL-02-5X group significantly increased by 1.34 -fold $(p=0.0003)$. The effect of PL-02 supplementation on serum lactate levels was also dose dependent.

Effect of PL-02 supplementation on fatigue-related biochemical parameters after the 10-min swim test or a 90 min swim test and 60 min rest 
As shown in Fig. 2a, after the 10 min swim test, the serum ammonia levels in the vehicle, PL-02-1X, PL-02$2 X$, and PL-02-5X groups were $166 \pm 24,150 \pm 21,138 \pm 37$, and $120 \pm 31 \mu \mathrm{mol} / \mathrm{L}$, respectively. Compared with vehicle group, PL-02-2X and PL-02-5X groups were significantly decreased by $16.58 \%(p=0.0423)$ and $27.85 \%(p=0.0011)$. The glucose levels in the vehicle, PL-02-1X, PL-02-2X, and PL-02-5X groups were $116 \pm 23,122 \pm 23,130 \pm 15$, and $135 \pm 17 \mathrm{mg} / \mathrm{dL}$. Only PL-02-5X group was significantly greater than vehicle group by 1.17 -fold $(p=0.0368)$ (Fig. $2 \mathrm{~b}$ ). Supplementation with PL-02 could decrease ammonia and improve glucose levels after exercise, both of which have a dose-dependent effect $(p<$ 0.0001).

The serum BUN levels were measured 60 min after the 90-min swimming test (Fig. 2c), which were found to be $38.6 \pm 2.1,33.1 \pm 2.5,31.4 \pm 5.0$, and $29.9 \pm 1.8 \mathrm{mg} / \mathrm{dL}$ in the mice in the vehicle, PL-02-1X, PL-02-2X, and PL-02-5X groups, respectively. Compared with the vehicle group, the PL-02-1X, PL-02-2X, and PL-02$5 X$ groups were significantly decreased by $14.42 \%(p=0.0003), 18.66 \%(p<0.0001)$, and $22.60 \%(p<$ $0.0001)$. The effect of PL-02 supplementation on serum BUN levels was also dose dependent $(p<$ $0.0001)$. An exercise injury index, $C K$, had a significant difference among the groups after the $90 \mathrm{~min}$ swim test and 60 min rest (Fig. 2d). Compared with the vehicle group, the PL-02-1X, PL-02-2X, and PL-02$5 \mathrm{X}$ groups were significantly decreased by $18.27 \%(p=0.0007), 21.63 \%(p<0.0001)$, and $22.67 \%(p<$ 0.0001). The effect of PL-02 supplementation on serum CK levels was also dose dependent $(p<0.0001)$.

\section{Effect of PL-02 Supplementation on liver and muscle glycogen}

The liver glycogen levels of the mice in the vehicle, PL-02-1X, PL-02-2X, and PL-02-5X groups were 20.44 $\pm 1.75,26.75 \pm 1.73,32.09 \pm 2.24 \mathrm{~m}$ and $34.58 \pm 1.88 \mathrm{mg} / \mathrm{g}$ liver, respectively (Fig. 3a). Compared with the vehicle group, the PL-02-1X, PL-02-2X, and PL-02-5X groups were significantly improved by 1.31 -fold ( $p<$ $0.0001), 1.57$-fold $(p<0.0001)$, and 1.69 -fold $(p<0.0001)$, respectively. Muscle glycogen levels in the vehicle, PL-02-1X, PL-02-2X, and PL-02-5X groups were $0.94 \pm 0.19,1.44 \pm 0.18,2.01 \pm 0.26 \mathrm{~m}$ and $2.11 \pm$ $0.15 \mathrm{mg} / \mathrm{g}$ muscle, respectively (Fig. 3b). The PL-02-1X, PL-02-2X, and PL-02-5X groups were significantly increased by 1.53 -fold $(p<0.0001), 2.14$-fold $(p<0.0001)$, and 2.24 -fold $(p<0.0001)$, respectively, as compared to the vehicle group. The effect of PL-02 supplementation on liver and muscle glycogen content was also dose dependent $(p<0.0001)$.

\section{Effect of PL-02 supplementation on biochemical profiles at the end of the study}

We assessed whether supplementation of PL-02 for four weeks had an impact on the health and safety and conducted biochemical parameter tests (Table 3). As a result, all biochemical parameters were within the normal range. In addition, there were no significant differences between the groups. Therefore, we believe that supplementation with the doses with PL-02 will not cause any damage. 
Table 3

Effects of PL-02 on biochemical parameters.

\begin{tabular}{|llllll|}
\hline Parameter & Vehicle & PL-02-1X & PL-02-2X & PL-02-5X & Trend Analysis \\
\hline AST (U/L) & $82 \pm 7$ & $81 \pm 8$ & $82 \pm 7$ & $82 \pm 6$ & 0.7425 \\
\hline ALT (U/L) & $57 \pm 18$ & $55 \pm 8$ & $55 \pm 8$ & $56 \pm 8$ & 0.0367 \\
\hline CK (U/L) & $563 \pm 74$ & $568 \pm 73$ & $564 \pm 38$ & $571 \pm 61$ & 0.7962 \\
\hline GLU (mg/dL) & $228 \pm 16$ & $228 \pm 38$ & $226 \pm 15$ & $227 \pm 51$ & 0.7189 \\
\hline CREA (mg/dL) & $0.39 \pm 0.01$ & $0.38 \pm 0.01$ & $0.38 \pm 0.01$ & $0.38 \pm 0.03$ & $0.0474 *$ \\
\hline BUN (mg/dL) & $22.4 \pm 2.5$ & $22.8 \pm 2.5$ & $22.3 \pm 2.8$ & $22.8 \pm 1.9$ & 0.6891 \\
\hline UA (mg/dL) & $1.3 \pm 0.3$ & $1.3 \pm 0.2$ & $1.3 \pm 0.3$ & $1.3 \pm 0.2$ & 0.5371 \\
\hline TC (mg/dL) & $152 \pm 8$ & $152 \pm 13$ & $152 \pm 20$ & $152 \pm 12$ & 0.9640 \\
\hline TG (mg/dL) & $157 \pm 21$ & $163 \pm 39$ & $156 \pm 12$ & $156 \pm 30$ & 0.9822 \\
\hline ALB (g/dL) & $3.3 \pm 0.1$ & $3.3 \pm 0.1$ & $3.3 \pm 0.1$ & $3.3 \pm 0.2$ & 0.6818 \\
\hline TP (g/dL) & $5.7 \pm 0.3$ & $5.7 \pm 0.2$ & $5.7 \pm 0.2$ & $5.7 \pm 0.3$ & 0.9014 \\
\hline $\begin{array}{l}\text { Data are expressed as mean } \pm \text { SD }(n=10 \text { mice per group). AST, aspartate aminotransferase; ALT, } \\
\text { alanine transaminase; CK, creatine kinase; GLU, glucose; CREA, creatinine; BUN, blood urea nitrogen; } \\
\text { UA, uric acid; TC, total cholesterol; TG, triacylglycerol; ALB, albumin; TP, total protein. }\end{array}$ \\
\hline
\end{tabular}

\section{Effect of PL-02 supplementation on tissue histology}

At the end of the study, we performed a histological evaluation of the liver, muscle, heart, kidney, lung, EFP, and BAT of the mice. As shown in Fig. 4, no abnormalities were observed in all groups. These results indicate that at the doses tested in this study, PL-02 has no adverse effects on organs and tissues.

\section{Effect of PL-02 Supplementation on gut microbiota}

At the end of the experiment, we analyzed the composition of the gut microbiota of mice treated with vehicle or PL-02 through 16S rRNA and observed the great changes in microbial ecology after PL-02 treatment. As shown on Fig. 5a, with the increased of the dose of PL-02, the percentage of Firmicutes in the gut microbiota also increased significantly. Among them, we could clearly see from the heat map that compared with the vehicle group (Fig. 5b), supplementation with PL-02 could effectively increase the proportion of Lactobacillus in the gut microbiota. In the Species level (Fig. 5c), with the increase in the dose of PL-02 supplements, the proportion of L. plantarum (Fig. $5 \mathrm{~d}$ ) in the gut microbiota increased significantly $(p<0.0001)$, and the abundance of the specific gravity of Akkermansia muciniphila (Fig. 5e) also increased significantly $(p<0.01)$. In particular, the human harmful gut microbiota, Blautia coccoides (Fig. 5f) and Pedobacter kwangyangensis (Fig. 5g), were significantly lower in the PL-02-treated group than in the vehicle group, and the percentage of hits were inversely proportional to dose increase $(p<$ 0.05). 


\section{Discussion}

In recent years, probiotics have been commonly used to promote health and improve body function, as well as for different benefits depending on the characteristics of the strain. In the current study, we screened the human-origin probiotic L. plantarum (PL-02) and designed different doses of probiotics to explore the benefits of improved exercise performance and anti-fatigue. We found that supplementing PL02 for four weeks can significantly increase the proportion of good bacteria in the intestine, muscle mass, muscle strength, endurance performance, and glycogen storage, and significantly reduce fatigue biochemical parameters after exercise, such as lactate, CK, and blood ammonia. In addition, it would not cause damage to healthy biochemical parameters and or cause tissue pathologies.

Supplementation with probiotics can effectively increase the a diversity in the intestinal and can also increase the production of SCFA in the intestine, thereby helping to maintain or restore the homeostasis of the intestine, promote health, and prevent many diseases ${ }^{29}$. Many studies have explored the crosstalk path between the gut and muscles and have found that the composition and interaction environment of the flora may affect the quality, function, and energy metabolism of the muscles by changing the flora, which is the so-called gut-muscle axis ${ }^{30,31}$. Among them, the metabolites of the gut microbiota may be involved in stimulating muscle energy metabolism and enhancing endurance performance, such as acetic acid $^{32}$. Acetic acid enhances glucose uptake and fatty acid metabolism by activating the activated protein kinase (AMPK) and increasing the expression of glucose transporter type 4 (GLUT4) and myoglobin. The myocyte enhancer factor 2A (MEF2A), which is a well-known transcription factor involved in the expression of myoglobin and GLUT4 genes, and found in the treat cell.Therefore, it is considered an important metabolic pathway for increasing muscle mass ${ }^{33}$. In previous studies, Supplementing heatinactivated Bifidobacterium breve B-3 for four consecutive weeks in rats found significant increases in muscle strength and mass, cytochrome $\mathrm{C}$ oxidase (CCO) gene expression, peroxisome proliferatoractivated receptor gamma coactivator (PGC) - $1 \mathrm{a}$ and phosphorylated AMPK in muscle ${ }^{34}$. In addition, previous study had shown that, 6-week supplementation of L. plantarum from kimchi has been found to significantly increase muscle mass in mice and humans ${ }^{22,23}$. These results seem to confirm that in this study, supplementation with human-origin L. plantarum also has significant benefits on improved muscle mass (Table 1) and strength (Fig. 1a and 1b).

On the other hand, propionic acid in SCFA can promote gluconeogenesis in liver cells ${ }^{35}$, while butyrate can maintain blood glucose homeostasis and promote glycogen metabolism through the GPR43-AKTGSK3 signaling pathway ${ }^{36}$. In a previous study, it was shown that probiotics (Lactobacillus acidophilus) regulate glycogen synthesis-related genes (GSK-3 $\beta$ and Akt) and glycogen content in tissues ${ }^{37}$. In a previous study, researchers explored the correlation between the composition of the gut microbiota and the content of muscle glycogen. The results showed that germ-free mice had lower muscle glycogen levels compared to individuals with a normal microbiome composition ${ }^{38}$. The glycogen content in the liver and muscles is a key factor in determining the aerobic energy metabolism of athletes. Disturbances in glycogen levels may lead to insufficient energy supply and decreased muscle strength and function, 
leading to impaired bioenergy metabolism ${ }^{39}$. Optimizing and increasing glycogen storage can effectively improve exercise endurance performance, thereby delaying fatigue after exercise and accelerating recovery ${ }^{40}$. In addition, previous studies have confirmed that supplementation with probiotics can significantly increase SCFA and fatty acid oxidation and activate peroxisome proliferator-activated receptor $y$ coactivator $1 a$ (PGC-1a), increasing ATP production, providing the energy needed for exercise and improving endurance exercise performance ${ }^{41}$. In our study, 4-week supplementation with $L$. plantarum PL-02 not only increased glycogen storage (Fig. 3), but also improved endurance exercise performance (Fig. 1c).

Similar to lactate, blood ammonia, BUN concentration, and CK activity would increase with the increasing time and intensity of exercise and would gradually recover with rest. Therefore, in many studies related to exercise and fatigue, they were often used as an important basis for judging peripheral fatigue caused by exercise $^{25}$. Lactate was the product of glycolysis in the anaerobic energy system and was accompanied by the production of hydrogen ions. With the increase in hydrogen ion concentration, the decrease in $\mathrm{pH}$ in blood and muscle tissue would cause muscle contraction and glycolysis to be inhibited, which could easily lead to muscle damage and decreased exercise capacity. In addition, in the process of ATP resynthesis, when AMP deaminase (AMPD) deaminates adenosine monophosphate (AMP) to inosine monophosphate (IMP), ammonia is produced and accumulated in skeletal muscle. Ammonia could be metabolized into BUN through the urea cycle ${ }^{28}$. However, probiotics could improve the production of blood lactate during exercise and be converted into SCFA, especially propionic acid and butyric acid, as well as speeding up the conversion of butyric acid into acetyl-CoA, which is used to generate ATP in the Krebs cycle and then provide the energy needed during exercise ${ }^{42}$. On the other hand, probiotics could also reduce intestinal permeability and inhibit bacterial urease activity to reduce ammonia in the blood ${ }^{43}$. Therefore, these results seemed to be validated in our research. We found that supplementation with PL02 for four consecutive weeks could not only reduce the blood lactate concentration and increase the rate of recovered after exercise, but also accelerate the elimination and recovery of blood lactic acid (Table 2) and reduced blood ammonia, BUN, and CK after exercise (Fig. 2a, 2c and 2d).

Through the 16S RNA analysis, we explored the distribution of gut microbiota by supplementation with $L$. plantarum (PL-02). The result showed that human-origin PL-02 could effectively and successfully colonize the gut of mice, as well as change the distribution ratio of the gut microbiota, which also seemed to play a role in energy absorption and regulation. A previous study showed that the core function of $L$. plantarum concerns carbohydrate metabolism and amino acid metabolism and provides molecular support for the strain to metabolize various sugars. Genes related to carbohydrate absorption and metabolism were closely related to genes encoding glycosidase and transporters ${ }^{44}$. Therefore, in our results it could be seen that as the feeding dose increases, the proportion of L. plantarum detected in the intestinal tract also increases significantly (Fig. 5d). In addition, we also found that compared with the placebo group, supplementation with PL-02 can significantly increase the proportion of Akkermansia muciniphila in the intestine (Fig. 5e). Previous studies have pointed out the higher proportion of the $A$. muciniphila genus in athletes' intestines, compared with sedentary people. They could maintain the 
intestinal barrier function and glucose homeostasis and played a role when endurance exercise was impaired. Therefore, $A$. muciniphila is positively correlated with exercise performance ${ }^{45}$. In addition, the supplementation of PL-02 increases the proportion of L. plantarum in the intestine and also effectively reduces the proportion of Bhatia coccids and Pedobacter kwangyangensis. According to a previous study, Blautia, especially $B$. coccoides, may activate the secretion of tumor necrosis factor- $a$ (TNF- $a$ ) and cytokines to a greater extent. Blautia is the most representative genus in type 2 diabetes patients and in prediabetes patients and is significantly higher than in patients with normal glucose tolerance ${ }^{46}$. According to another animal experiment, the abundance of Pedobacter kwangyangensis in the gut of mice on a high-calorie diet was greatly increased. Perhaps the abundance of this strain is related to obesity ${ }^{47}$. In the current study, we believe that supplementation with L. plantarum PL-02 for four consecutive weeks would not cause any physiological, biochemical, or histopathological hazards.

\section{Declarations}

\section{Declarations}

\section{Conflicts}

of Interest:

\section{Funding:}

This study was funded by projects from the university-industry cooperation fund (NTSU No.1091027), National Taiwan Sport University, Taoyuan, Taiwan.

\section{Author Contributions:}

M.-C.L., H.-H.H. and C.-C.H. designed the study. M.-C.L., H.-H.H., W.-Y.L., Y.-W.K., S.-Y.T. and Y.-J.H. carried out the experiments. H.-H.H, W.-Y.L., Y.-W.K., S.-Y.T., W.-L.C., C.-L.L. and C.-C.H. provided reagents and other laboratory supplies. M.-C.L., H.-H.H., Y.-J.H., and C.-C.H. analyzed the data. M.-C.L., Y.-W.K. and C.-C.H. prepared figures and wrote the manuscript. M.-C.L., C.-L.L. and C.-C.H. revised the manuscript.

\section{Acknowledgments:}

The authors are grateful to coach Wei-Ling Chen for providing her valuable gut microbiota. We thank Chin-Shan Ho for assistance in measuring forelimb grip strength and Bioflag Biotech Co., Ltd. (Tainan, Taiwan) for providing the probiotics.

\section{References}


1. Davis, J. M. \& Bailey, S.P. Possible mechanisms of central nervous system fatigue during exercise. Med Sci Sports Exerc. 29(1), 45-57 (1997).

2. Wan, J. J., Qin, Z., Wang, P. Y., Sun, Y. \& Liu, X. Muscle fatigue: general understanding and treatment. Exp Mol Med. 49(10), e384 (2017).

3. Gonzalez, J. T., Fuchs, C. J., Betts, J. A. \& van Loon, L. J. Liver glycogen metabolism during and after prolonged endurance-type exercise. Am J Physiol Endocrinol Metab. 311(3), E543-553 (2016).

4. Burnley, M. \& Jones, A. M. Power-duration relationship: Physiology, fatigue, and the limits of human performance. Eur J Sport Sci. 18(1), 1-12 (2018).

5. Finsterer, J. Biomarkers of peripheral muscle fatigue during exercise. BMC Musculoskelet Disord. 13, 218 (2012).

6. Kim, J. H. et al. Anti-Fatigue Effect of Prunus Mume Vinegar in High-Intensity Exercised Rats. Nutrients 12(5), 1205 (2020).

7. Donati Zeppa, S. et al. Mutual Interactions among Exercise, Sport Supplements and Microbiota. Nutrients 12(1), 17 (2019).

8. Mitchell, C. M. et al. Does Exercise Alter Gut Microbial Composition? A Systematic Review. Med Sci Sports Exerc. 51(1), 160-167 (2019).

9. Zhang, Z., Tang, H., Chen, P., Xie, H. \& Tao, Y. Demystifying the manipulation of host immunity, metabolism, and extraintestinal tumors by the gut microbiome. Signal Transduct Target Ther. 4, 41 (2019).

10. Hsu, Y. J. et al. Effect of intestinal microbiota on exercise performance in mice. J Strength Cond Res. 29(2), 552-558 (2015).

11. Conlon, M. A. \& Bird, A. R. The impact of diet and lifestyle on gut microbiota and human health. Nutrients 7(1), 17-44 (2014).

12. Donohoe, D. R. et al. The microbiome and butyrate regulate energy metabolism and autophagy in the mammalian colon. Cell Metab. 13(5), 517-526 (2011).

13. Gao, Z. et al. Butyrate improves insulin sensitivity and increases energy expenditure in mice. Diabetes. 58(7), 1509-1517 (2009).

14. Mollica, M. P et al. Butyrate Regulates Liver Mitochondrial Function, Efficiency, and Dynamics in Insulin-Resistant Obese Mice. Diabetes 66(5), 1405-1418 (2017).

15. Ticinesi, A. et al. Exercise and immune system as modulators of intestinal microbiome: implications for the gut-muscle axis hypothesis. Exerc Immunol Rev. 25, 84-95 (2019).

16. Zhou, Y. et al. Exploration of bacterial community classes in major human habitats. Genome Biol. 2014, 15(5), R66.

17. Derrien, M. \& van Hylckama Vlieg, J. E. Fate, activity, and impact of ingested bacteria within the human gut microbiota. Trends Microbiol. 23(6), 354-366 (2015).

18. Parvez, S., Malik, K. A., Ah Kang, S. \& Kim, H. Y. Probiotics and their fermented food products are beneficial for health. J App/ Microbiol. 100(6), 1171-1185 (2006). 
19. Brandi, J.et al. Exploring the wound healing, anti-inflammatory, anti-pathogenic and proteomic effects of lactic acid bacteria on keratinocytes. Sci Rep. 10(1), 11572 (2020).

20. Lee, C. S. \& Kim, S. H. Anti-inflammatory and Anti-osteoporotic Potential of Lactobacillus plantarum A41 and L. fermentum SRK414 as Probiotics. Probiotics Antimicrob Proteins. 12(2), 623-634 (2020).

21. Duncan, S. H., Louis, P. \& Flint, H. J. Lactate-utilizing bacteria, isolated from human feces, that produce butyrate as a major fermentation product. Appl Environ Microbiol. 70(10), 5810-5817 (2004).

22. Chen, Y. M. et al. Lactobacillus plantarum TWK10 Supplementation Improves Exercise Performance and Increases Muscle Mass in Mice. Nutrients 8(4), 205 (2016).

23. Huang, W. C. et al. Effect of Lactobacillus plantarum TWK10 on Exercise Physiological Adaptation, Performance, and Body Composition in Healthy Humans. Nutrients 11(11), 2836 (2019).

24. Maughan, R. J. et al. IOC consensus statement: dietary supplements and the high-performance athlete. Br J Sports Med. 52(7), 439-455 (2018).

25. Huang, W. C. et al. Exercise Training Combined with Bifidobacterium longum OLP-01 Supplementation Improves Exercise Physiological Adaption and Performance. Nutrients 12(4), 1145 (2020).

26. Huang, W. C. et al. The Effects of Thiamine Tetrahydrofurfuryl Disulfide on Physiological Adaption and Exercise Performance Improvement. Nutrients. 10(7), 851 (2018).

27. Lee, M. C. et al. Lactobacillus salivarius Subspecies salicinius SA-03 is a New Probiotic Capable of Enhancing Exercise Performance and Decreasing Fatigue. Microorganisms 8(4), 545 (2020).

28. Lee, M. C. et al. In Vivo Ergogenic Properties of the Bifidobacterium longum OLP-01 Isolated from a Weightlifting Gold Medalist. Nutrients 11(9), 2003 (2019).

29. Nagpal, R. et al. Human-origin probiotic cocktail increases short-chain fatty acid production via modulation of mice and human gut microbiome. Sci Rep. 8(1), 12649 (2018).

30. Grosicki, G. J., Fielding, R. A. \& Lustgarten, M. S. Gut Microbiota Contribute to Age-Related Changes in Skeletal Muscle Size, Composition, and Function: Biological Basis for a Gut-Muscle Axis. Calcif Tissue Int. 102(4), 433-442 (2018).

31. Ticinesi, A. et al. Gut Microbiota, Muscle Mass and Function in Aging: A Focus on Physical Frailty and Sarcopenia. Nutrients 11(7), 1633 (2019).

32. Toda, K. et al. Heat-Killed Bifidobacteriumbreve B-3 Enhances Muscle Functions: Possible Involvement of Increases in Muscle Mass and Mitochondrial Biogenesis. Nutrients. 12(1):219 (2020).

33. Maruta, H. et al. Activation of AMP-Activated Protein Kinase and Stimulation of Energy Metabolism by Acetic Acid in L6 Myotube Cells. PLoS One. 11(6), e0158055 (2016).

34. Toda, K. et al. Heat-Killed Bifidobacteriumbreve B-3 Enhances Muscle Functions: Possible Involvement of Increases in Muscle Mass and Mitochondrial Biogenesis. Nutrients 12(1), 219 (2020).

35. Samuel, B. S. et al. Effects of the gut microbiota on host adiposity are modulated by the short-chain fatty-acid binding G protein-coupled receptor, Gpr41. Proc Natl Acad Sci U S A. 105(43), 16767- 
$16772(2008)$.

36. Zhang, W. Q. et al. Sodium Butyrate Improves Liver Glycogen Metabolism in Type 2 Diabetes Mellitus. J Agric Food Chem. 67(27), 7694-7705 (2019).

37. Yan, F. et al. Lactobacillus acidophilus alleviates type 2 diabetes by regulating hepatic glucose, lipid metabolism and gut microbiota in mice. Food Funct. 10(9), 5804-5815 (2019).

38. Nay, K. et al. Gut bacteria are critical for optimal muscle function: a potential link with glucose homeostasis. Am J Physiol Endocrinol Metab. 317(1), E158-E171 (2019).

39. Philp, A., Hargreaves, M. \& Baar, K. More than a store: regulatory roles for glycogen in skeletal muscle adaptation to exercise. Am J Physiol Endocrinol Metab. 302(11), E1343-E1351 (2012).

40. Lawler, T. P. \& Cialdella-Kam, L. Non-carbohydrate Dietary Factors and Their Influence on PostExercise Glycogen Storage: a Review. Curr Nutr Rep. 9(4), 394-404 (2020).

41. Clark, A. \& Mach, N. The Crosstalk between the Gut Microbiota and Mitochondria during Exercise. Front Physiol. 8, 319 (2017).

42. Scheiman, J. et al. Meta-omics analysis of elite athletes identifies a performance-enhancing microbe that functions via lactate metabolism. Nat Med. 25(7), 1104-1109 (2019).

43. Bongaerts, G., Severijnen, R. \& Timmerman, H. Effect of antibiotics, prebiotics and probiotics in treatment for hepatic encephalopathy. Med Hypotheses. 64(1), 64-68 (2005).

44. Mao, B. et al. Comparative Genomic Analysis of Lactiplantibacillus plantarum Isolated from Different Niches. Genes (Basel) 12(2), 241 (2021).

45. Barton, W. et al. The microbiome of professional athletes differs from that of more sedentary subjects in composition and particularly at the functional metabolic level. Gut. 67(4), 625-633 (2018).

46. Egshatyan, L. et al. Gut microbiota and diet in patients with different glucose tolerance. Endocr Connect. 5(1), 1-9 (2016).

47. Tung, Y. C. et al. Piceatannol Exerts Anti-Obesity Effects in C57BL/ 6 Mice through Modulating Adipogenic Proteins and Gut Microbiota. Molecules 21(11), 1419 (2016).

\section{Figures}

(a)

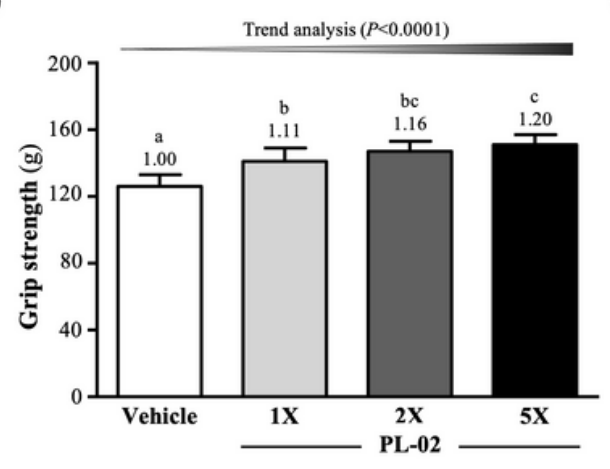

(b)

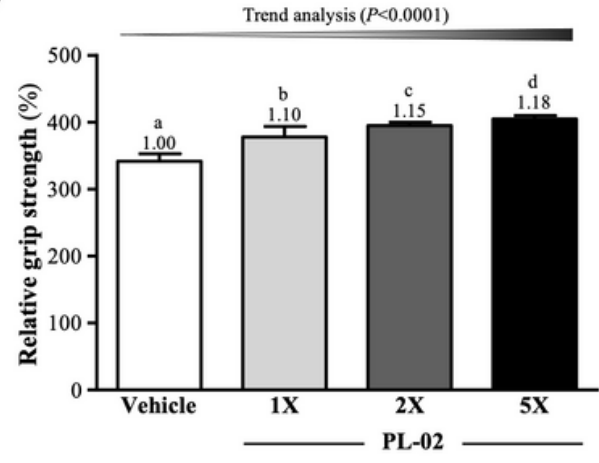

(c)

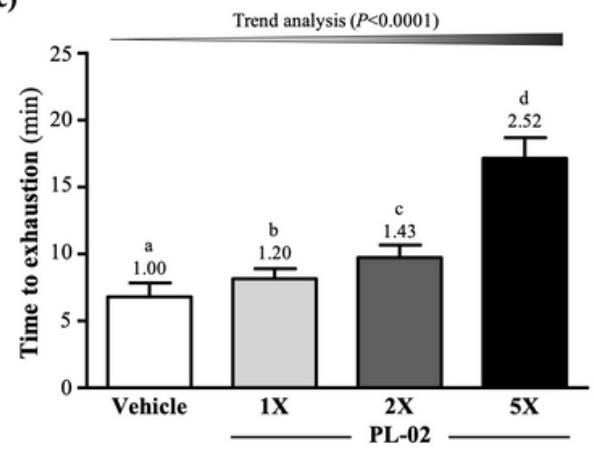


Figure 1

Effect of 4 weeks of PL-02 supplementation on (a) absolute forelimb grip strength, (b) forelimb grip strength (\%) relative to body weight and (c) exhaustive swim time. Data are expressed as mean \pm SD for $n$ $=10$ mice per group. Different superscript letters $(a, b, c, d)$ indicate significant difference at $p<0.05$.

(a)

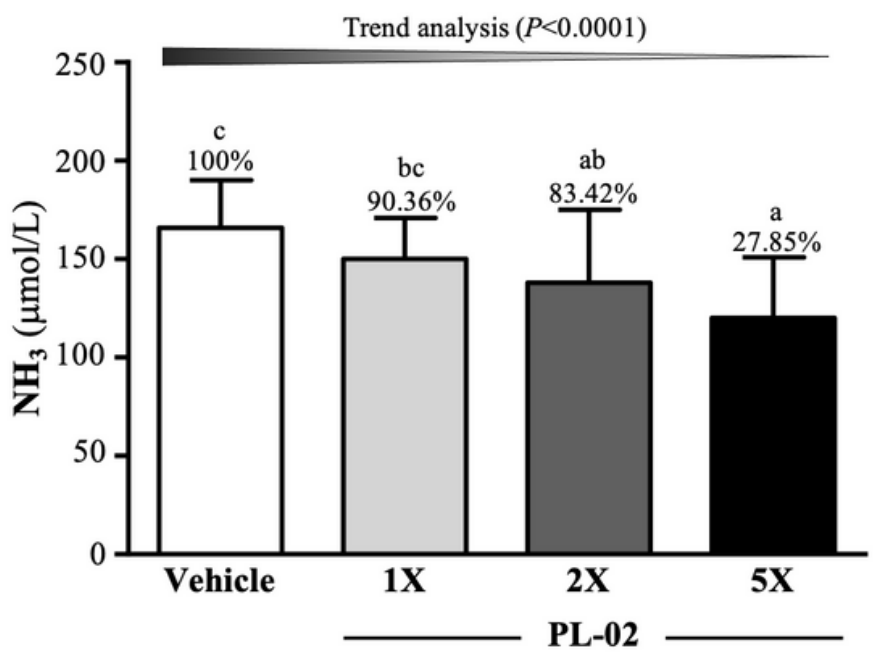

(c)

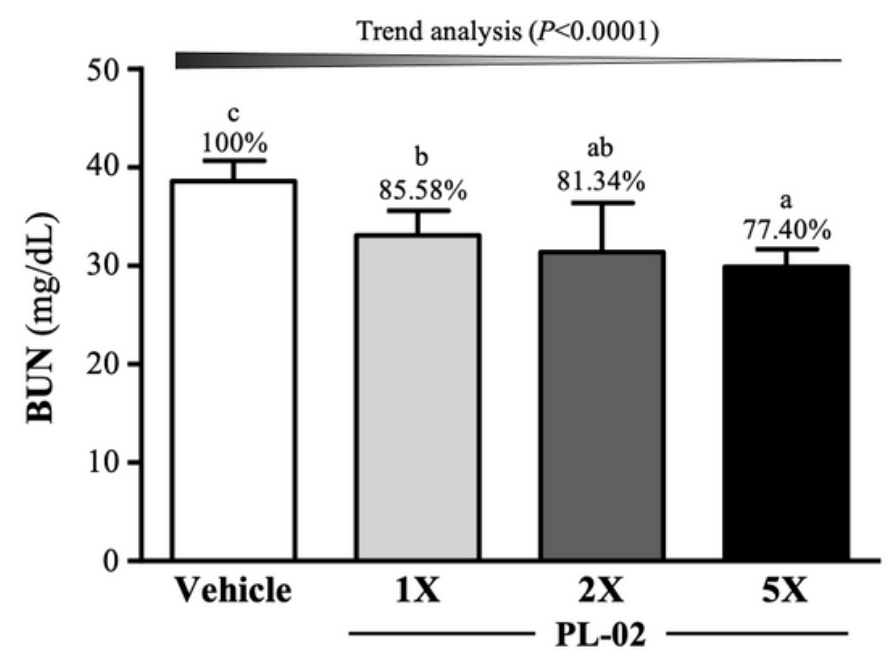

(b)

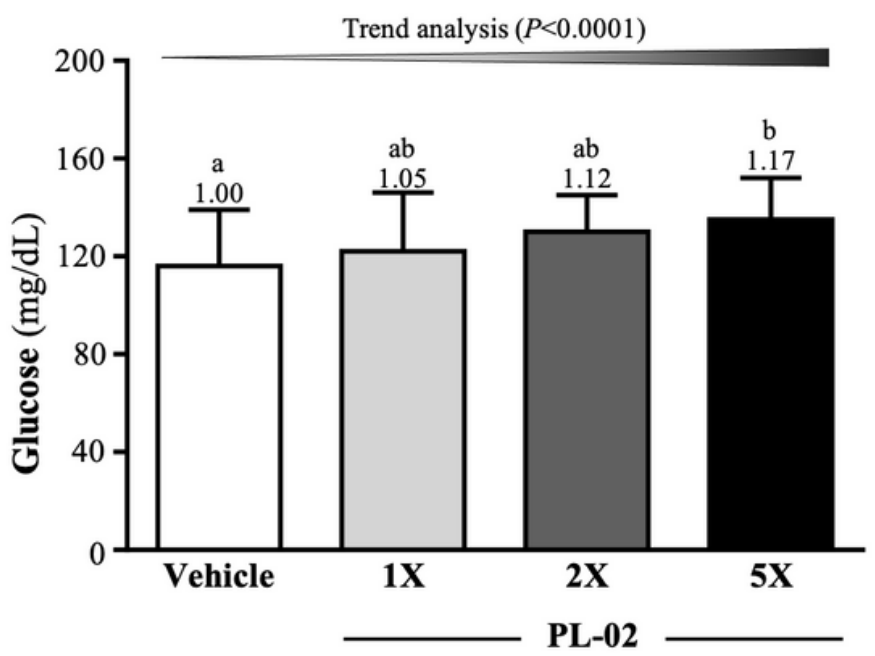

(d)

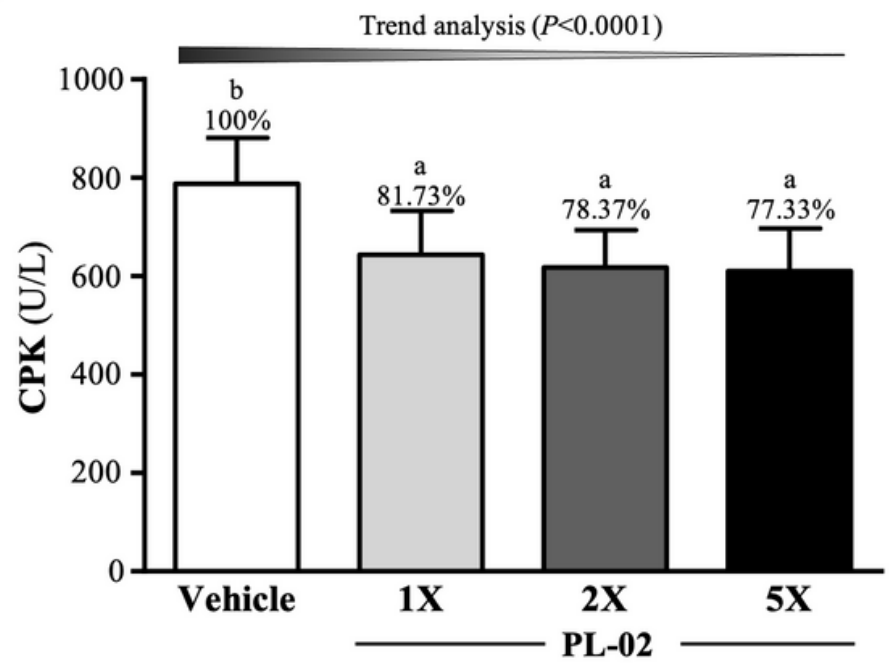

Figure 2

Effect of 4 weeks PL-02 supplementation on (a) NH3 and (b) glucose, (c) BUN and (d) CK. Data are expressed as mean \pm SD for $n=10$ mice per group. Different superscript letters $(a, b, c)$ indicate significant difference at $p<0.05$. 
(a)

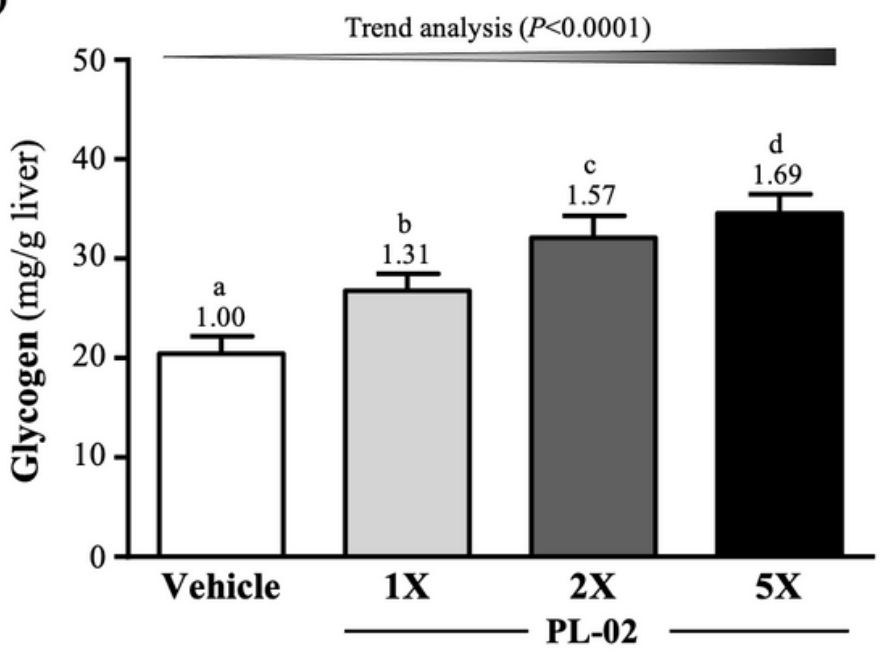

(b)

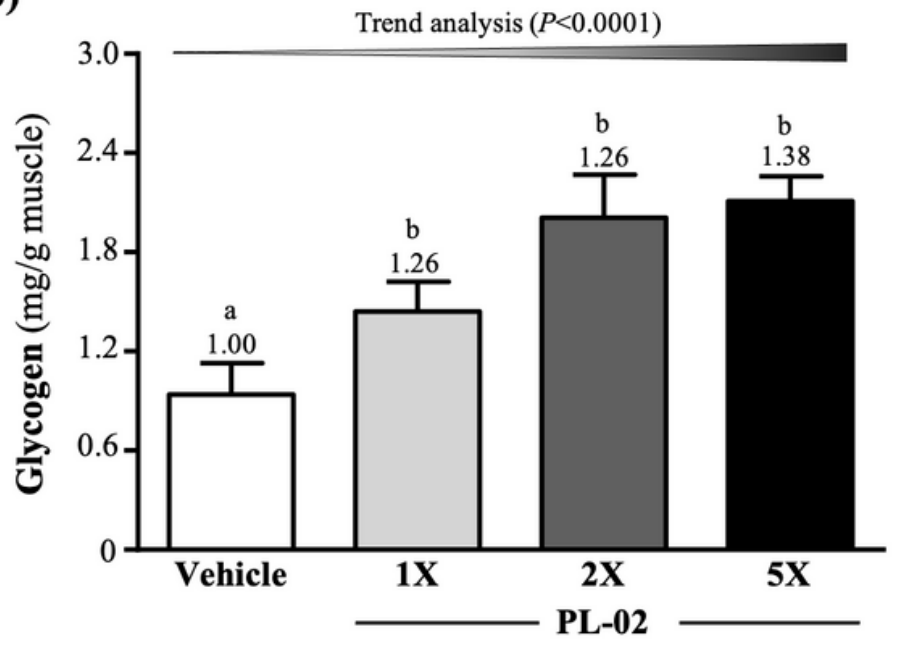

Figure 3

Effect of 4 weeks PL-02 supplementation on (a) liver glycogen and (b) muscle glycogen. Data are expressed as mean \pm SD for $n=10$ mice per group. Different superscript letters $(a, b, c, d)$ indicate significant difference at $p<0.05$. 
(a)

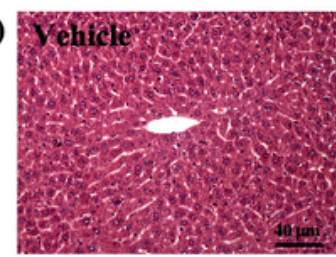

(b)

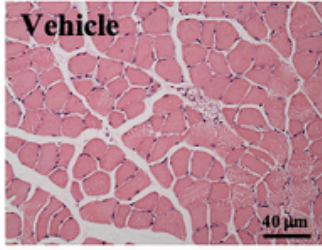

(c)

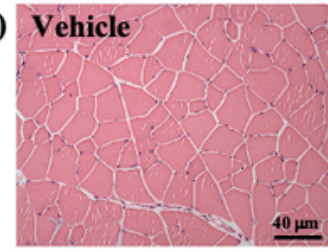

(d)

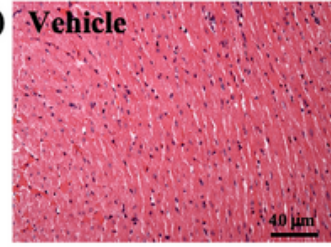

(e)

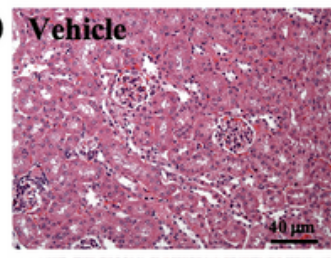

(f)

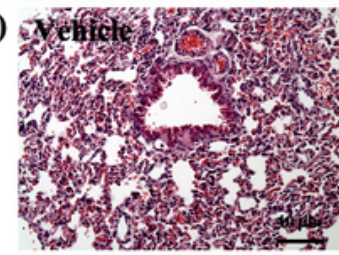

(g)

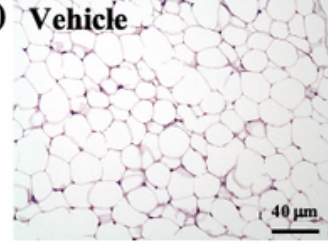

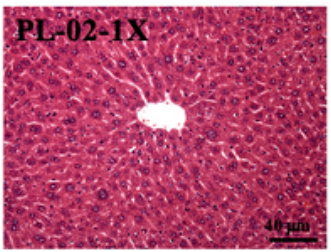
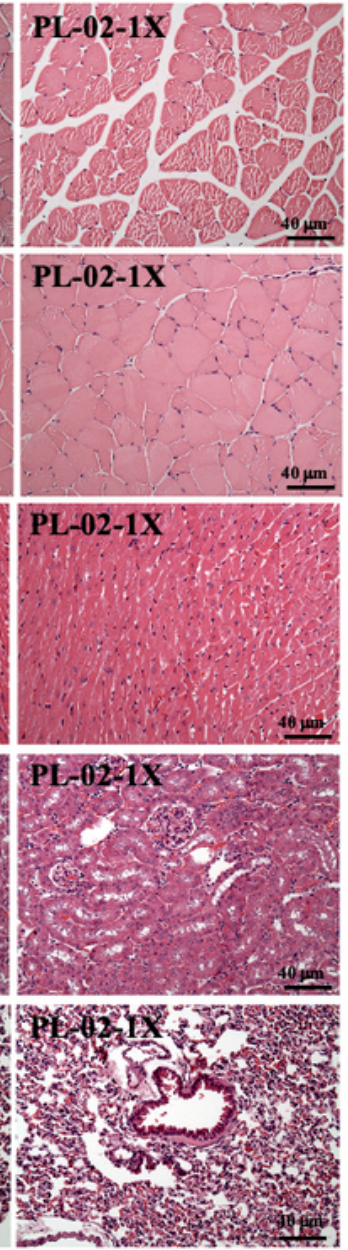

PL-02-1X

(h) Vehicte $P L 02-1 X$

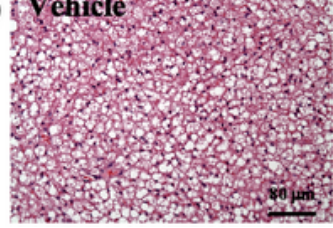

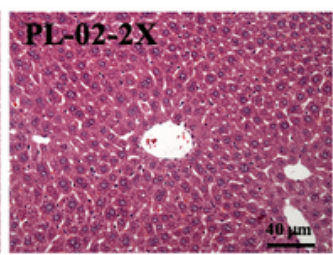
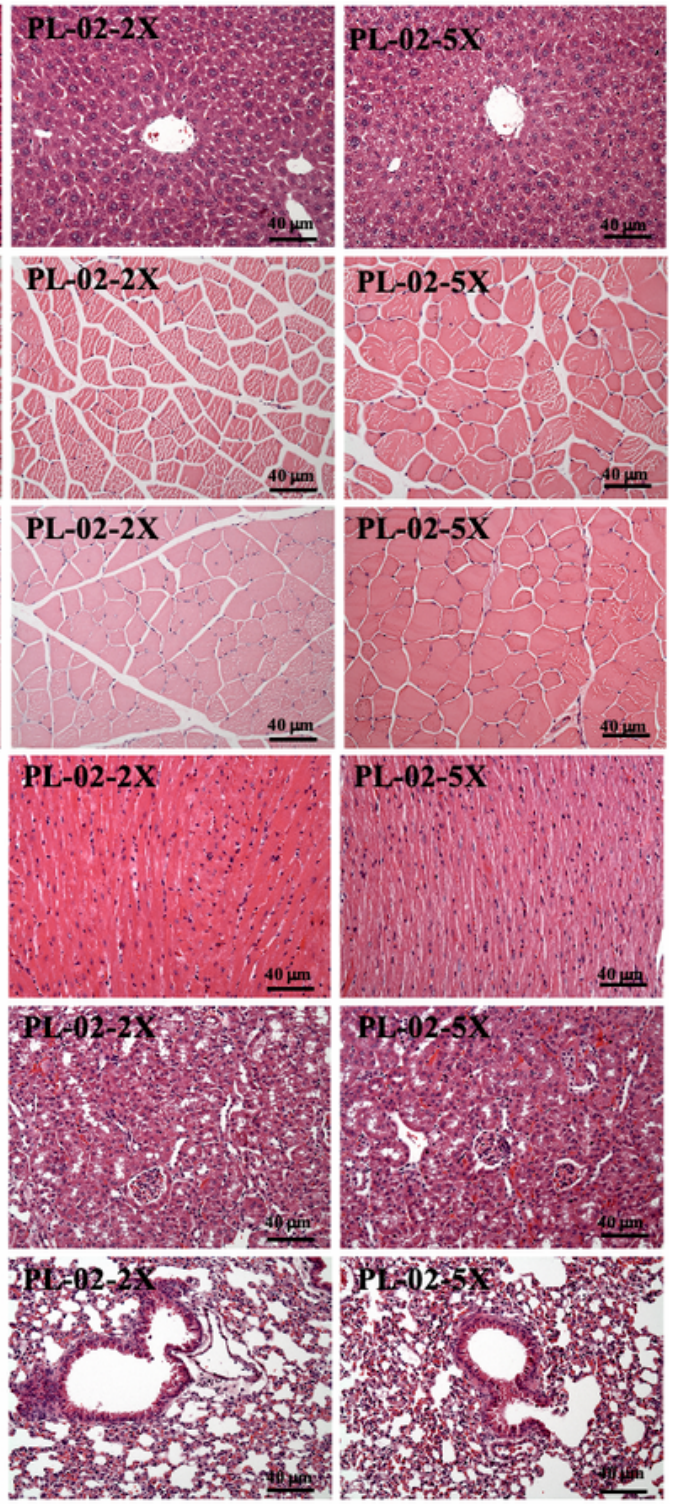

PL-02-2X

PL-02-5X

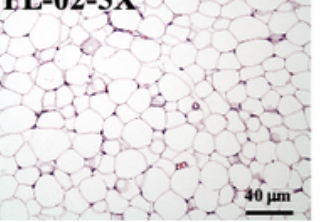

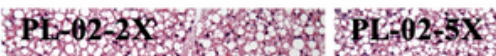
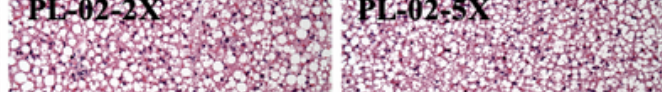

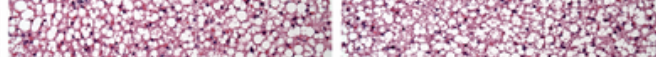

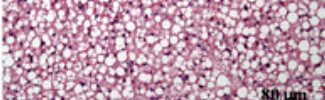

(6)

\section{Figure 4}

Effect of PL-02 supplementation on (a) liver, (b) muscle, (c) quadricep muscles, (d) heart, (e) kidney, (f) lung, (g) adipocyte tissue, and (h) BAT tissue in mice. (H\&E stain, magnification: 200x; bar, $40 \mu \mathrm{m}$; BAT magnification: 100x; bar, $80 \mu \mathrm{m})$. 
(a)

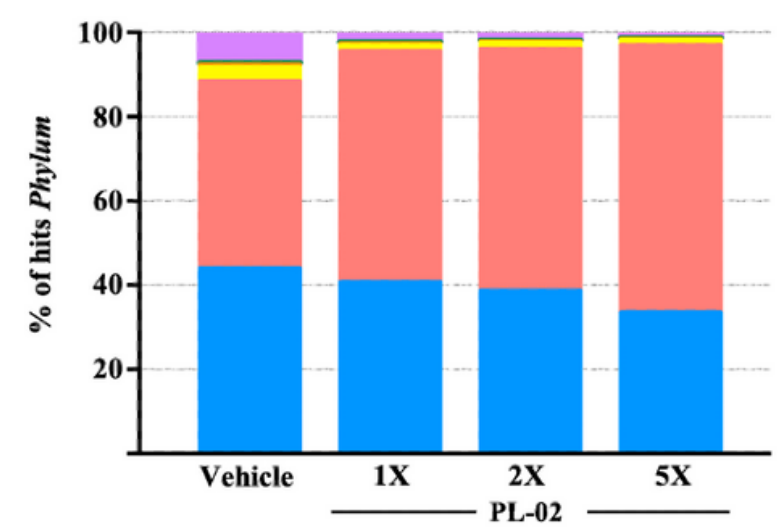

(b)

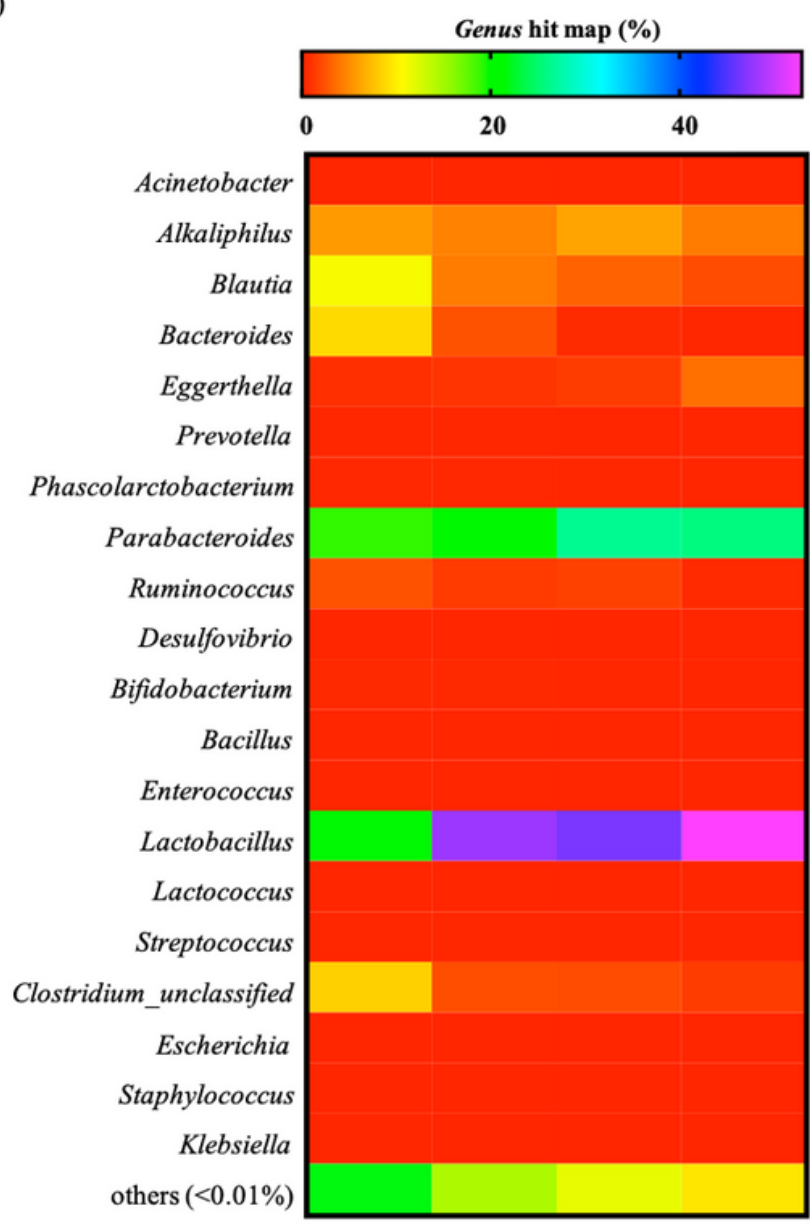

(c)

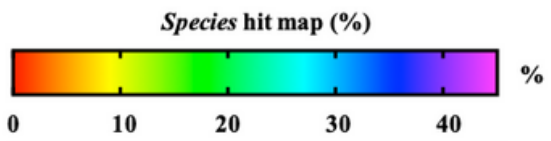

Adlercreutzia equolifaciens Alkaliphilus crotonatoxidans

Bacteroides acidifaciens Blautia coccoides Blautia wexlerae

Dysgonomonas wimpennyi Erysipelothrix muris Faecalibacterium prausnitzii Lachnospira pectinoschiza Megamonas funiformis Mucispirillum schaedleri Parabacteroides goldsteini Roseburia faecis

Ruminococcus gnavus Oribacterium sinus

Akkermansia muciniphila Bifidobacterium adolescentis Lactobacillus hayakitensis Lactobacillus intestinalis Lactobacillus plantarum Bacteroides fragilis

Bacteroides stercoris

Clostridium alkalicellulosi Clostridium thermosuccinogenes Escherichia coli Pedobacter kwangyangensis Staphylococcus aureus Others $<0.01 \%$

(d)

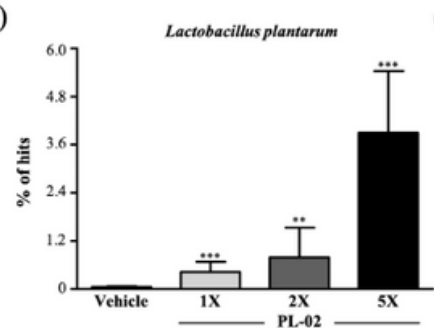

(f)

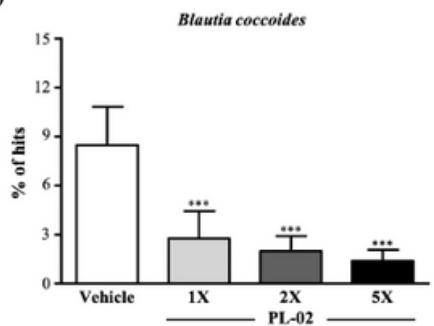

(e)

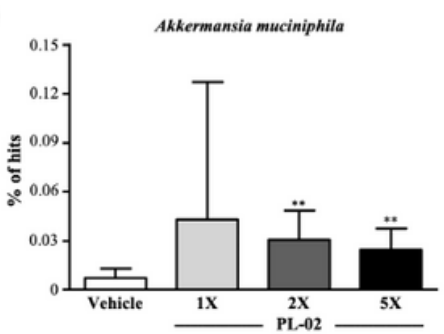

(g)

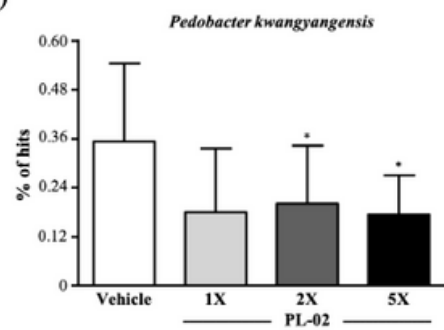

\section{Figure 5}

Effect of PL-02 supplementation on (a) phylum, (b) genus, (c) species, (d) Lactobacillus plantarum \% hits, (e) Akkermansia muciniphila. \% hits, (f) Blautia coccoides \% hits, and (g) Pedobacter kwangyangensis \% hits. Data are expressed as mean $\pm S D$ for $n=8$ mice per group. Values with different superscript letters are significantly different at $* p<0.05 ; * \star p<0.01 ; * \star \star p<0.0001$. 